\title{
Variations in the Antithyroid Antibody Titre During Pregnancy and After Delivery
}

This article was published in the following Dove Press journal:

Risk Management and Healthcare Policy

\author{
Chuyu Li $\mathbb{D}^{1-3, *}$ \\ Na Zhang ${ }^{1-3, *}$ \\ Jing Zhou ${ }^{1-3}$ \\ Wingting Leung ${ }^{1-3}$ \\ Hans-Jürgen Gober ${ }^{4}$ \\ Zengshu Huang ${ }^{1-3}$ \\ Xinyao Pan $\left(\mathbb{D}^{1-3}\right.$ \\ Lijia Chen ${ }^{1-3}$ \\ Liang Guan ${ }^{5,6}$ \\ Ling Wang (1D ${ }^{1-3}$
}

\begin{abstract}
'Laboratory for Reproductive Immunology, Hospital \& Institute of Obstetrics and Gynecology, Shanghai Medical College, Fudan University, Shanghai, People's Republic of China; ${ }^{2}$ The Academy of Integrative Medicine of Fudan University, Shanghai, People's Republic of China; ${ }^{3}$ Shanghai Key Laboratory of Female Reproductive Endocrine-Related Diseases, Shanghai, People's Republic of China; ${ }^{4}$ Department of Pharmacy, Neuromed Campus, Kepler University Hospital, Linz, 4020, Austria; ${ }^{5}$ Department of Nuclear Medicine, Ruijin Hospital, School of Medicine, Shanghai Jiaotong University, Shanghai, People's Republic of China; ${ }^{6}$ Department of Nuclear Medicine, Ruijin Hospital North, School of Medicine, Shanghai Jiaotong University, Shanghai, People's Republic of China
\end{abstract}

*These authors contributed equally to this work

Correspondence: Liang Guan Ruijin Hospital of Shanghai Jiaotong

University, 197 Ruijin 2nd Road, Huangpu

District, Shanghai, 200025, People's Republic

of China

Tel +8618917762612

Fax + 86-2I-6433 3548

Email drliangguan@outlook.com

Ling Wang

Obstetrics \& Gynecology Hospital of Fudan University, 4 I 9 Fangxie Road, Shanghai,

2000 I I, People's Republic of China

$\mathrm{Tel}+86-|822| 8|5| 82$

Fax + 86-02I-6345733I

Email Dr.wangling@fudan.edu.cn
Background: Immunosuppression occurs during pregnancy, and the antithyroid antibody titre drops, rebounding after delivery. We aimed to determine variations in antithyroid antibody titres during pregnancy and after delivery.

Methods: This retrospective study was conducted in a single centre. Antibody titres of 142 patients were measured to assess variations in the levels of thyroid-stimulating hormone receptor antibodies (TRAbs), thyroid peroxidase antibodies (TPOAbs), and thyroid globulin antibodies (TgAbs). We compared the titres of each antibody between adjacent time periods (eg, first trimester (T1) vs second trimester (T2), T2 vs third trimester (T3), T3 vs the postpartum period (PP)) by paired $t$-test or the Wilcoxon test. Then, we analysed data from patients with complete laboratory examination results in all four periods with the Friedman test, performing comparisons among groups.

Results: In the TgAb group, significant differences existed between $\mathrm{T} 1$ and $\mathrm{T} 2$ and between T2 and T3 in the LT4 subgroup and between T1 and T2 in the no-medication subgroup. In the TRAb group, significant differences existed between T1 and T2 in the LT4 subgroup. In the TPOAb group, significant differences existed among each group in the LT4 subgroup, and there were significant differences between T1 and T2 and between T2 and T3 in the nomedication subgroup. The Friedman test showed that the P-values were 0.013 and 0.004 in the LT4 and no-medication subgroups of the TgAb group, respectively; 0.122 in the LT4 subgroup of the TRAb group; and $<0.001$ and 0.272 in the LT4 and no-medication subgroups of the TPOAb group, respectively. In the LT4 subgroup of the TgAb group, the P-values for comparisons of time periods were 0.602 between $\mathrm{T} 1$ and T2, 0.602 between $\mathrm{T} 2$ and $\mathrm{T} 3$, 0.006 between $\mathrm{T} 1$ and T3, and 0.602 between T3 and PP. In the no-medication subgroup of the TgAb group, the P-values were 0.078 between T1 and T2, 1.000 between T2 and T3, 0.011 between T1 and T3, and 0.078 between T3 and PP. In the LT4 subgroup of the TPOAb group, the P-values were 0.09 between $\mathrm{T} 1$ and T2, 0.014 between $\mathrm{T} 2$ and $\mathrm{T} 3,<0.001$ between $\mathrm{T} 1$ and $\mathrm{T} 3$, and 0.772 between $\mathrm{T} 3$ and PP.

Conclusion: We can conclude that the $\mathrm{TgAb}$ and TPOAb titres dropped during pregnancy. Keywords: autoimmune thyroid disease, thyroid-stimulating hormone receptor antibody, thyroid peroxidase antibody, thyroid globulin antibody

\section{Introduction}

Approximately $10 \%$ of the general population suffers from autoimmune thyroid diseases (AITDs), and at least 2-3\% of pregnant women are affected by thyroid dysfunction worldwide. ${ }^{1}$ When women become pregnant, they undergo many physiological changes and may face diseases, influencing the health of both the mother and foetus. The associations among antithyroid antibodies, thyroid diseases, and pregnancy have garnered increasing attention. Three kinds of antithyroid 
antibodies, namely, thyroid-stimulating hormone receptor antibodies (TRAbs), thyroid peroxidase antibodies (TPOAbs), and thyroid globulin antibodies (TgAbs), are closely associated with AITDs, regardless of pregnancy status. Some studies have reported that 10 to $15 \%$ of the healthy population are positive for TPOAbs and TgAbs and that clinical hypothyroidism may develop from subclinical hypothyroidism in $2 \%$ of the healthy population. The TPOAb-positive rate varies with age, race and ethnicity, and the positive rate of TgAbs is lower than that of TPOAbs in Graves' disease (GD) patients. ${ }^{2,3}$ While abnormal increases in TgAbs and TPOAbs can also be detected in healthy subjects, abnormal increases in TRAbs can be found only in the sera of most GD patients and in 10-15\% of patients who have Hashimoto's thyroiditis. ${ }^{4}$

According to the guidelines in China and America, these antibodies should be monitored in patients with certain conditions. For example, according to the 2017 Guidelines of the American Thyroid Association for the Diagnosis and Management of Thyroid Disease During Pregnancy and the Postpartum Period, if the concentration of TRAb increases in early pregnancy, testing should be repeated at weeks 18-22 (strong recommendation, moderate-quality evidence). According to the 2012 Guidelines of the Chinese Society of Endocrinology and Chinese Society of Perinatal Medicine for the Diagnosis and Management of Thyroid Disease During Pregnancy and the Postpartum Period, the measurement of TRAbs between 20 and 24 weeks of pregnancy in GD patients or patients who previously had GD is recommended since the titre of TRAbs can help doctors assess the pregnancy outcome (recommendation rank B). Thus, according to the guidelines, we can infer that the monitoring of those antibodies is very important.

Recently, several studies reported an association between abnormal increases in antithyroid antibodies during and after pregnancy and pathologies, such as pregnancy loss associated with TRAbs; ${ }^{5-7}$ preterm delivery, postpartum depression (PPD), foetal or neonatal thyrotoxicosis associated with TPOAbs; ${ }^{8-10}$ and foetal brain development outcomes associated with TgAbs. ${ }^{11}$ Methods for treating abnormal increases in these antibodies have also received attention. Dhillon-Smith et al did not find significant differences in the rates of live births with the use of $50 \mu \mathrm{g}$ levothyroxine (LT4) once daily among euthyroid women with TPOAbs. ${ }^{12}$ Nazarpour et al found that replacement therapy with LT4 in pregnant women with thyroidstimulating hormone $(\mathrm{TSH})$ concentrations $\geq 4.0 \mathrm{mIU} / \mathrm{L}$ was beneficial in reducing the probability of preterm delivery. ${ }^{13}$ Therefore, it is important to determine how to prevent the occurrence of AITDs and identify treatment methods that can be used during pregnancy.

Moreover, an increasing number of researchers have gradually found a changing pattern in which the titre of antithyroid antibodies will drop during pregnancy and rebound after delivery. De Leo et al identified the typical pattern of change in TRAbs, ${ }^{6}$ and the variation in TRAbs during pregnancy and after delivery has also been reported recently. ${ }^{4,16}$ In addition to variations in TRAbs, variations in both TPOAbs and TgAbs have been reported. ${ }^{3,17,18}$ However, to date, no study has included all antibody types. In our study, we explored variations in the three antibody types during pregnancy and after delivery. To this end, we analysed the variations in antibody titres during and after pregnancy instead of measuring the positive rates. Additionally, we implemented different methods to analyse these antibody variations. Notably, these three characteristics have not been reflected in other articles.

\section{Materials and Methods Study Design}

This is a retrospective study in a single centre. We divided our research into two parts to explore the variations in antibodies during pregnancy and after delivery.

\section{Subjects}

We collected data from 142 pregnant Chinese women who consulted with Dr. Liang Guan as their attending doctor between January 1, 2000, and December 31, 2020, at Ruijin Hospital. The inclusion criteria were women who had an antithyroid antibody test (TPOAb, TgAb, or TRAb) in Ruijin Hospital during pregnancy or after delivery. Since necessary examinations were different for each patient, not all patients had tests for all three antibodies (TRAb, TPOAb and $\operatorname{TgAb}$ ). Thus, the titre data of those antibodies were divided into three groups according to the antibody type. The examination time span was separated into 4 periods: from date of the last menstrual period to $12^{+6}$ weeks as the first period (T1), from $13^{+0}$ weeks to $28^{+6}$ weeks as the second period (T2), from $29^{+0}$ weeks to the date of delivery (T3), and from the date of delivery to 12 weeks after delivery as the postpartum period (PP). We calculated the average antibody titre in each period for each patient. Not all patients had data for adjacent periods. These cases 
were excluded per our exclusion criterion (36 in the TgAb group, 47 in the TRAb group, and 34 in the TPOAb group). As a result, the TgAb group included 37 patients, the TRAb group included 19 patients, and the TPOAB group included 45 patients (Figure 1). Then, we divided patients into subgroups according to the medication used (PTU, LT4, or no medication). Finally, there were 4, 17, and 16 patients in the PTU, LT4 and no-medication subgroups, respectively, of the TgAb group; 4, 8, and 7 patients in PTU, LT4 and nomedication subgroups, respectively, of the TRAb group; and 6, 26, and 13 patients in the PTU, LT4 and nomedication subgroups, respectively, of TPOAb group. The basic patient information for each subgroup is presented in Table 1. We obtained informed consent from each study participant prior to study commencement. The study was approved by the Ethics Committee of the Obstetrics and Gynecology Hospital of Fudan University (Kyy2018-85), and the study was conducted in accordance with the Declaration of Helsinki.

\section{Methods}

The serum levels of TSH, FT3, FT4, TgAb, and TPOAb were tested by automated chemiluminescent immunoassays (Architect i2000SR; Abbott Laboratories, Chicago, IL). The functional sensitivity of serum TSH was $0.0036 \mathrm{mIU} /$ L. The laboratory reference ranges provided by the manufacturer are as follows: TSH, 0.35-4.94 mIU/L; FT4, 9.01-$19.05 \mathrm{pmol} / \mathrm{L}$; FT3, 2.63-5.70 pmol/L; TPOAb, < $5.61 \mathrm{IU} /$ $\mathrm{mL}$; and TgAb, $<4.11 \mathrm{IU} / \mathrm{mL}$. The serum levels of TRAb were measured by electrochemiluminescence immunoassays (Cobas 601 analyzer, Roche Diagnostics) with a suggested cut-off value of $1.75 \mathrm{IU} / \mathrm{L}$

\section{Statistical Analysis}

The statistical analysis was performed using SPSS 25.0 (IBM SPSS, Armonk, NY USA), and graphs were drawn using GraphPad Prism 8.0.2 (USA, GraphPad Software). Since a patient might have had multiple tests for a particular antibody in one period, we first calculated the average antibody titre for each patient. Then, we compared antibody titres between adjacent periods (T1 vs T2, T2 vs T3, T3 vs PP) in the LT4 and no-medication subgroups of each group by paired $t$-test or the Wilcoxon test. Finally, we selected patients with complete laboratory examination results in all four periods and used the Friedman test and groupwise comparisons. Two-tailed $\mathrm{P}<0.05$ was considered statistically significant.

\section{Results}

In the first part of the data analysis, the numbers of pairs between T1 and T2, T2 and T3, and T3 and PP were 12, 10 , and 9, respectively, in the LT4 subgroup of the TgAb group and 12, 11, and 9, respectively, in the no-medication subgroup of the $\mathrm{TgAb}$ group. P-values were 0.0029 , 0.0367 , and 0.1235 in the LT4 subgroup and 0.0061 , 0.1095 , and 0.0506 in the no-medication subgroup with the Wilcoxon test or paired $t$-test. In the TRAb group, the numbers of pairs between T1 and T2, T2 and T3, and T3 and PP were 8, 4, and 3, respectively, in the LT4 subgroup and 2, 3, and 4, respectively, in the no-medication subgroup. The P-values were $0.016,0.2348$, and 0.585 for $\mathrm{T} 1$ vs T2, T2 vs T3, and T3 vs PP, respectively, in the LT4 subgroup and 0.4892 and 0.1277 for T2 vs T3 and T3 vs $\mathrm{PP}$, respectively, in the no medication subgroup with the Wilcoxon test or paired $t$-test (the Wilcoxon test and paired $t$-test were not applicable for the 2 pairs of patients in the $\mathrm{T} 1 / \mathrm{T} 2$ comparison in the no-medication subgroup). In the $\mathrm{TPOAb}$ group, the numbers of pairs between $\mathrm{T} 1$ and $\mathrm{T} 2$, T2 and T3, and T3 and PP were 22, 21, and 16, respectively, in the LT4 subgroup and 8, 10, and 9, respectively, in the no-medication subgroup. The P-values for T1 vs T2, T2 vs T3, and T3 vs PP were $\mathrm{P}<0.0001,0.001$, and 0.0171 , respectively, in the LT4 subgroup and 0.0499, 0.0051, and 0.8169 , respectively, in the no-medication group with the Wilcoxon test or paired $t$-test (Figures 2-4).

In the second part of the data analysis, there were 4 and 7 people in the LT4 and no-medication subgroups, respectively, in the TgAb group. In the TRAb group, 3 people and 1 person were in the LT4 and no-medication subgroups, respectively, and in the TPOAb group, 13 and 4 people were in the LT4 and no-medication subgroups, respectively. We used the Friedman test, and the resulting P-values were 0.013 and 0.004 in the $\mathrm{TgAb}$ group, 0.122 in the TRAb group, and $\mathrm{P}<0.001$ and 0.272 in the TPOAb group (Table 2). Then, in the LT4 subgroup of the TgAb group, the P-values for comparisons among groups were 0.602 between $\mathrm{T} 1$ and T2, 0.602 between $\mathrm{T} 2$ and T3, 0.006 between T1 and T3, and 0.602 between T3 and PP. In the no-medication subgroup of $\mathrm{TgAb}$ group, the $\mathrm{P}$-values for comparisons among groups were 0.078 between $\mathrm{T} 1$ and T2, 1.000 between T2 and T3, 0.011 between $\mathrm{T} 1$ and T3, and 0.078 between T3 and PP. In the LT4 subgroup of the TPOAb group, the P-values for comparisons among groups were 0.09 between $\mathrm{T} 1$ and $\mathrm{T} 2$, 0.014 between $\mathrm{T} 2$ and T3, $\mathrm{P}<0.001$ between $\mathrm{T} 1$ and T3, and 0.772 between T3 and PP (Figure 5). 
In the TgAb group, 36 patients without adjacent data were excluded; in the TRAb group, 47 patients without adjacent data were excluded; and in the TPOAb group, 34 patients without adjacent data were excluded.
Figure I The flowchart of the experiment.

\section{Discussion}

In this study, we investigated the variations in antithyroid antibody titres during pregnancy and after delivery. It was found that the titres of both TgAbs and TPOAbs decreased during pregnancy, while only the titres of TPOAbs rebounded after delivery in patients with LT4 treatment.
142 patients who visited Ruijin Hospital and received antithyroid antibody laboratory examination were recruited for participation in this study.

According to the laboratory examination results of the patients, the average values of T1, T2, T3 and PP were obtained for each patient. According to the antibody types, the patients were divided into the TgAb group with 73 patients, the TRAb group with 66 patients, and the TPOAb group with 79 patients.

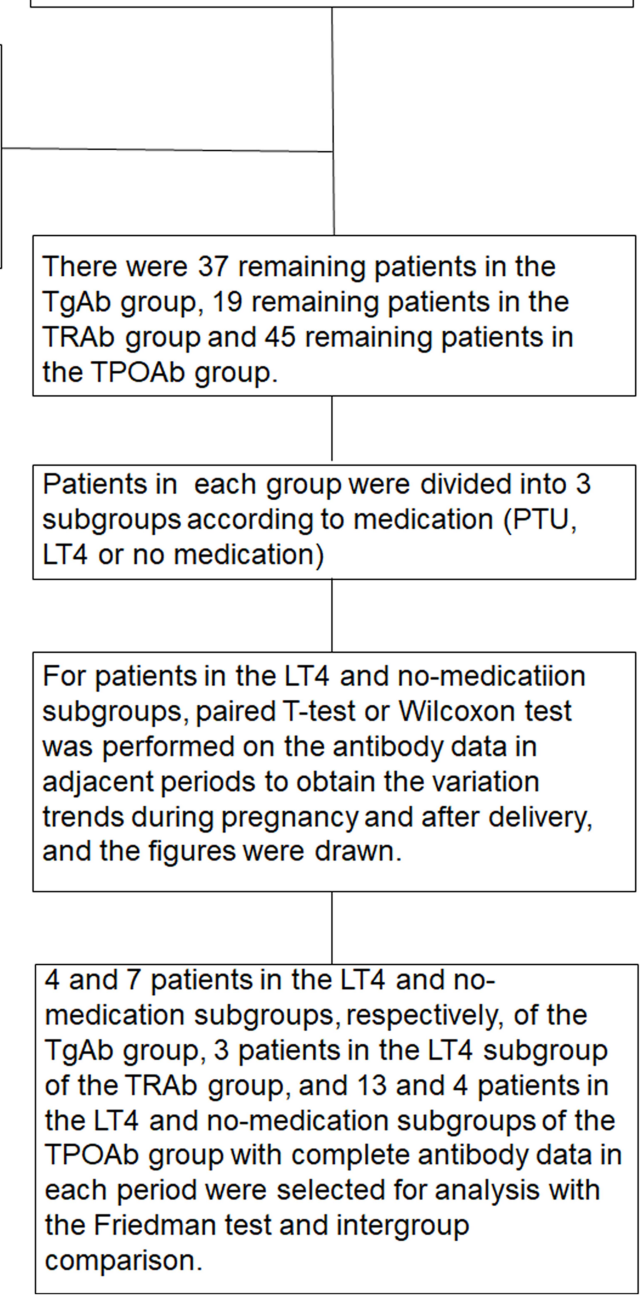

Previous researchers have shown that the levels of many different antibodies, including TgAbs, TPOAbs and TRAbs, may decrease during pregnancy and rebound after delivery. ${ }^{418-22}$ Regarding TgAbs, the study by Tamaki showed that the average TgAb level of 9 pregnant women in $\mathrm{T} 2$ was significantly lower than that in $\mathrm{T} 1(\mathrm{P}<0.01)$, and 


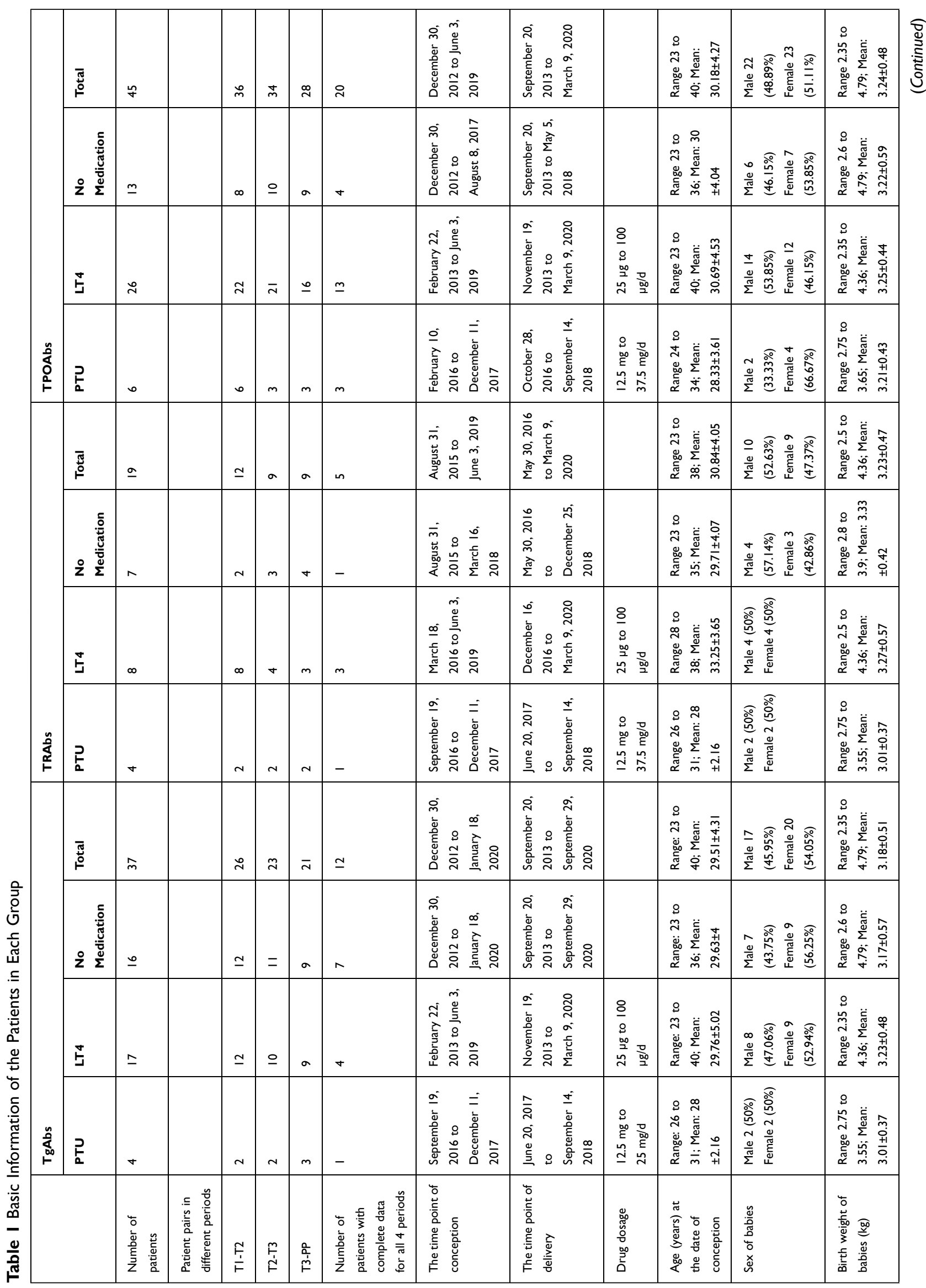




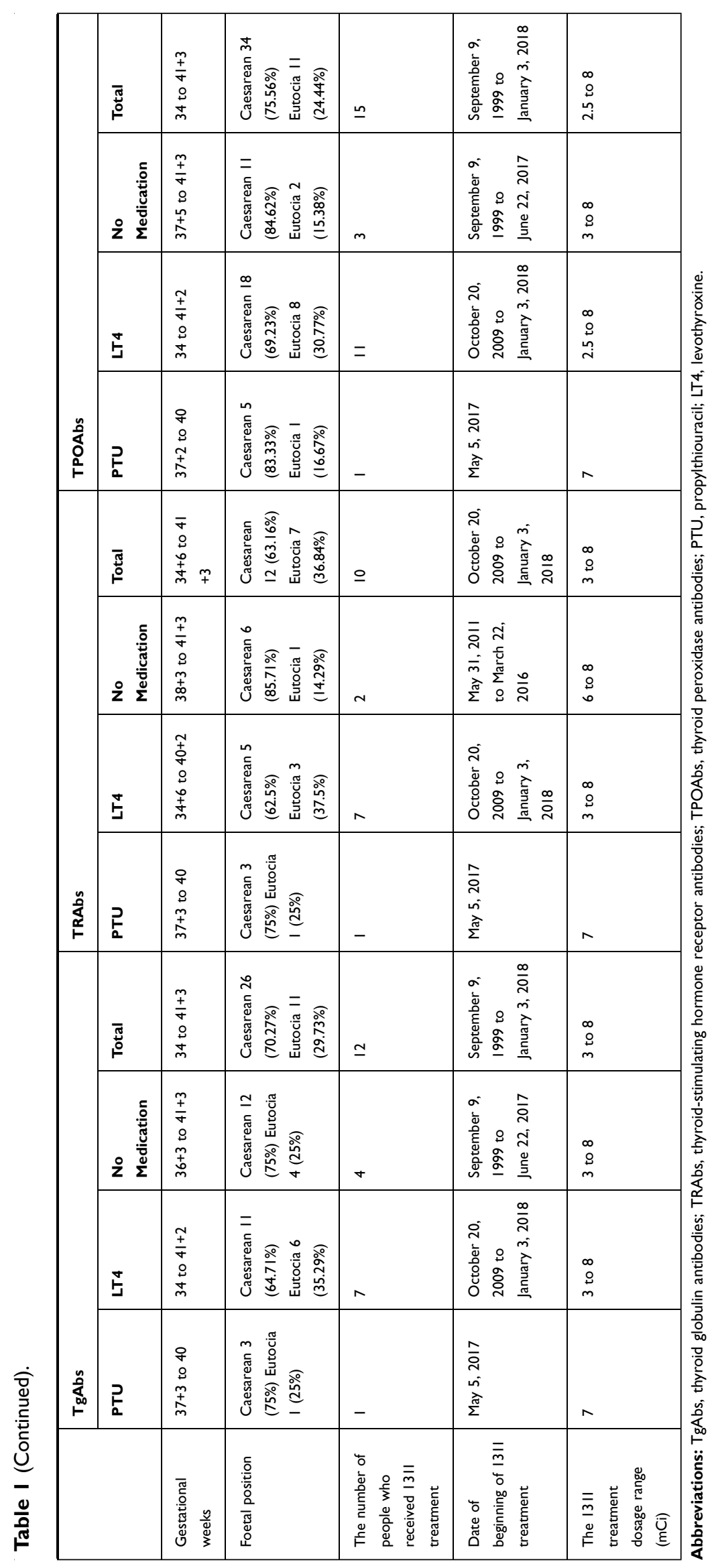



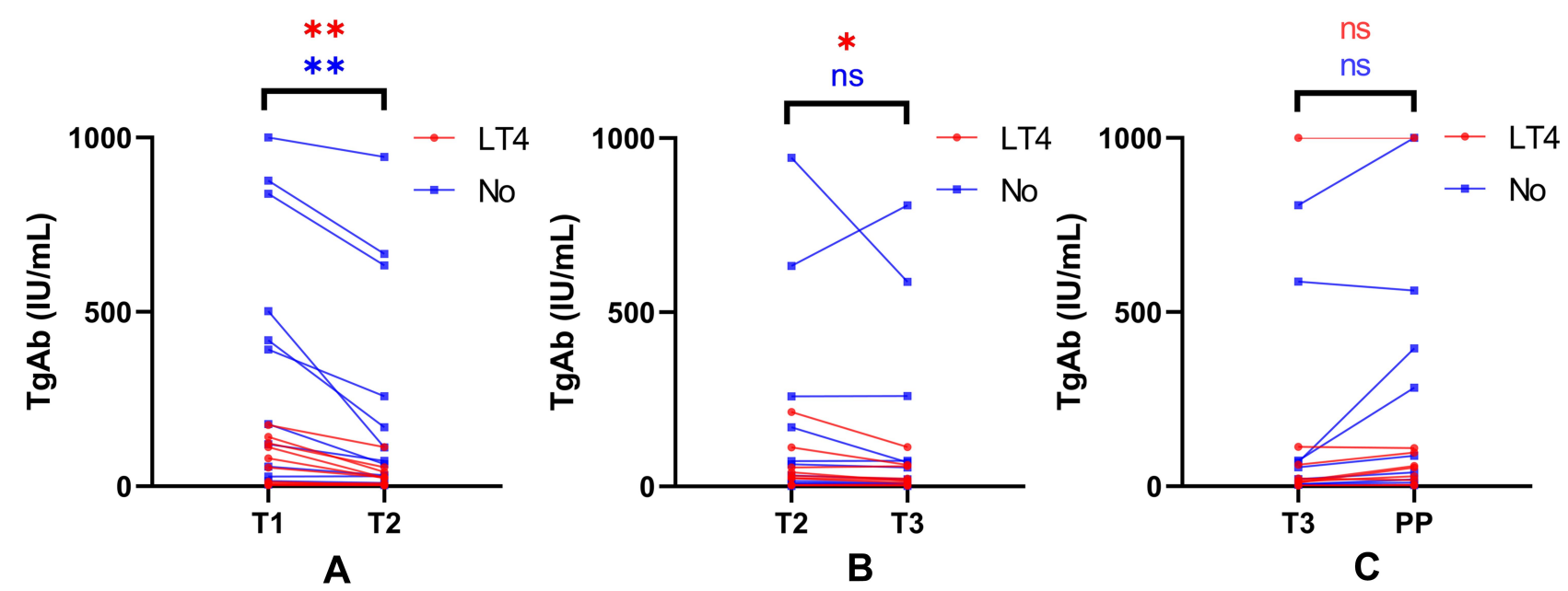

Figure 2 Comparison of TgAb titres in adjacent periods. The curves are a comparison of TgAbs in two adjacent periods. (A) Comparison between TI and T2 for 12 pairs of patients in the LT4 subgroup and 12 pairs of patients in the no-medication subgroup. (B) Comparison between T2 and T3 for 10 pairs of patients in the LT4 subgroup and II pairs of patients in the no-medication subgroup. (C) Comparison between T3 and PP for 9 pairs of patients in the LT4 subgroup and 9 pairs of patients in the nomedication subgroup. $* 0.01<\mathrm{P}<0.05 ; * * 0.00 \mathrm{I}<\mathrm{P} \leq 0.0 \mathrm{I}$.

Abbreviations: LT4, levothyroxine; No, no medication; ns, no significant difference.
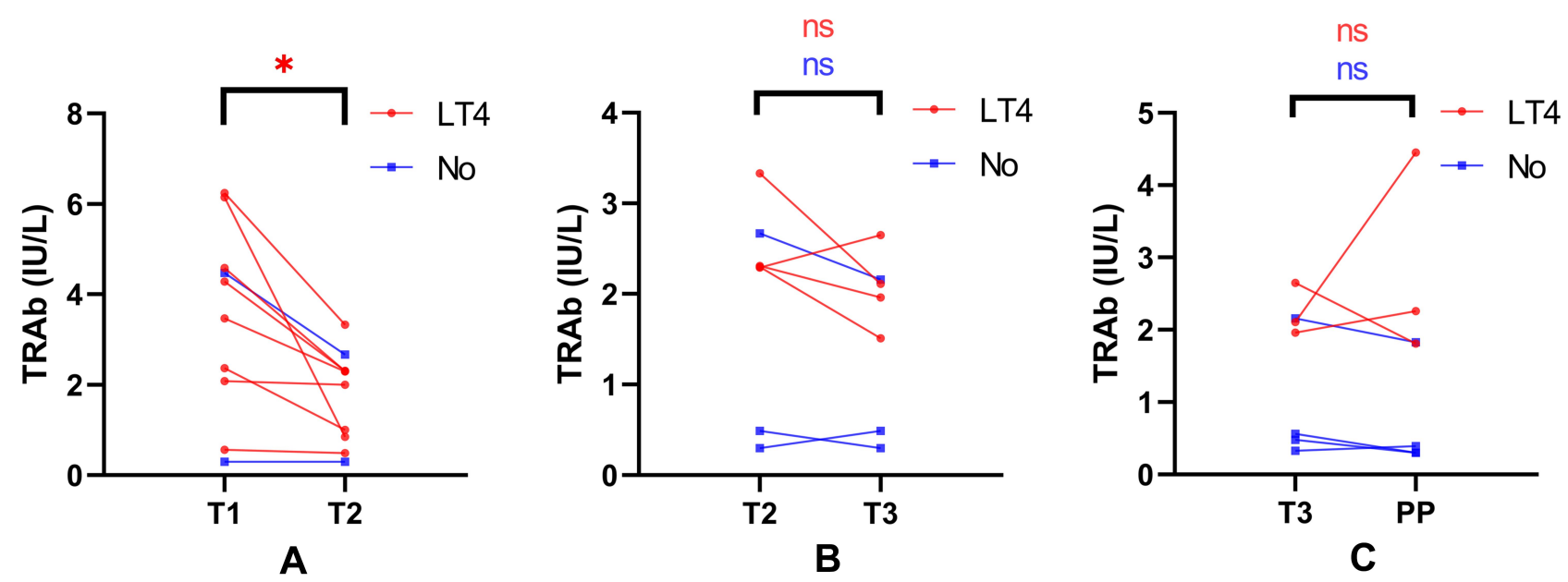

Figure 3 Comparison of TRAbs in adjacent periods. The curves are a comparison of TRAbs in two adjacent periods. (A) Comparison between TI and T2 for 8 pairs of patients in the LT4 subgroup and 2 pairs of patients in the no-medication subgroup. (B) Comparison between T2 and T3 for 4 pairs of patients in the LT4 subgroup and 3 pairs of patients in the no-medication subgroup. (C) Comparison between T3 and PP for 3 pairs of patients in the LT4 subgroup and 4 pairs of patients in the no-medication subgroup. $* 0.01<\mathrm{P}<0.05$.

Abbreviations: LT4, levothyroxine; No, no medication; ns, no significant difference.

the level in T3 was significantly lower than that in T2 $(\mathrm{P}<0.05)$. In addition, the level in the early postpartum stage (1-3 months postpartum) was significantly higher than that in the third trimester $(\mathrm{P}<0.001)$. Unfortunately, specific data were not provided by the authors. ${ }^{20}$ Instead of titre, several other studies reported a decrease in the positive rate of TgAbs in 25 women during pregnancy. P.P.A. Smyth et al found that the TgAb-positive rates were $36.8 \%, 12.0 \%$, $4.0 \%$ and $0 \%$ in the nonpregnant group and in the first, second and third trimesters of the pregnancy group, respectively. Six weeks postpartum, the $\mathrm{TgAb}$-positive rate of the pregnant group did not change compared with that in the third trimester $(0 \%){ }^{23}$ According to the analysis in the first part of our study, the antibody titres dropped significantly during pregnancy in both the LT4 and no-medication subgroups of the $\operatorname{TgAb}$ group. In the second part of the study, significant differences also appeared between the T1 and T3 periods in both the LT4 and no-medication subgroups of the $\mathrm{TgAb}$ group. However, the rebound in antibody titre after delivery did not appear in either subgroup in 

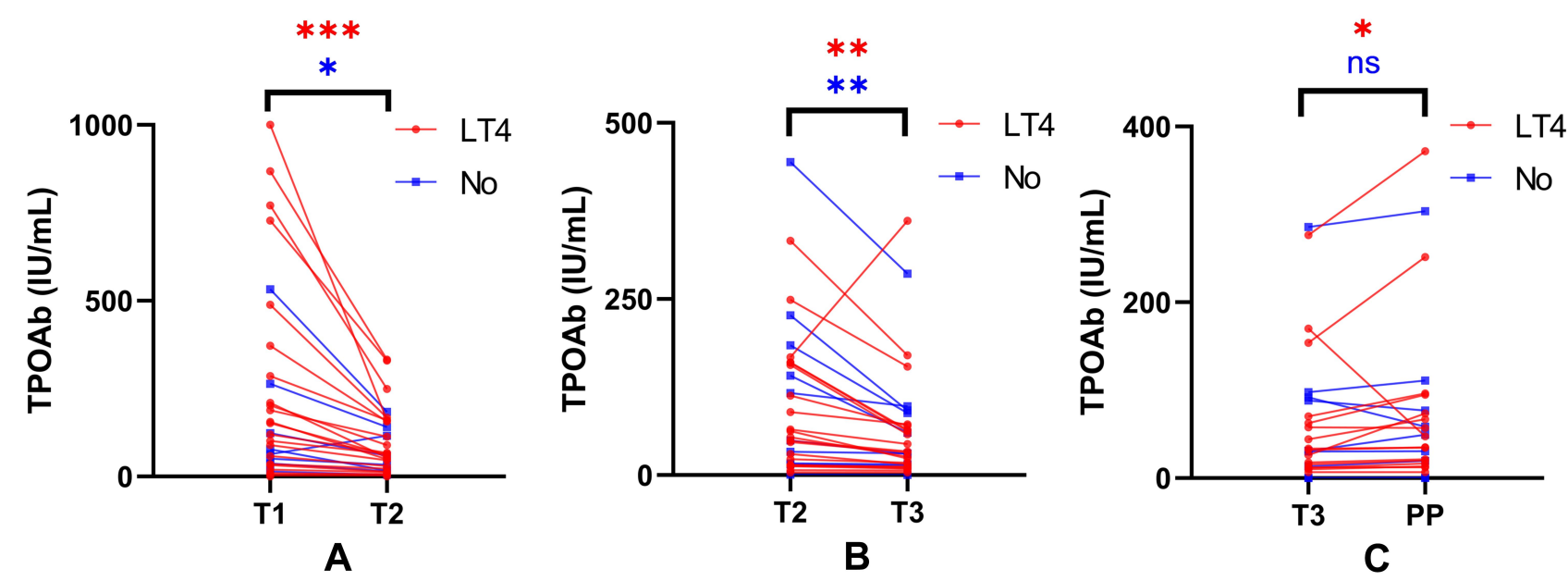

Figure 4 Comparison of TPOAbs in adjacent periods. The curves are a comparison of TPOAbs in two adjacent periods. (A) Comparison between TI and T2 for 22 pairs of patients in the LT4 subgroup and 8 pairs of patients in the no-medication subgroup. (B) Comparison between T2 and T3 for 21 pairs of patients in the LT4 subgroup and 10 pairs of patients in the no-medication subgroup. (C) Comparison between T3 and PP for 16 pairs of patients in the LT4 subgroup and 9 pairs of patients in the no-medication subgroup. $* 0.01<\mathrm{P}<0.05 ; * * 0.00 \mathrm{I}<\mathrm{P} \leq 0.01 ; * * * \mathrm{P} \leq 0.00 \mathrm{I}$.

Abbreviations: LT4, levothyroxine; No, no medication; ns, no significant difference.

either part of our analysis. We infer that 3 months was too short for the $\mathrm{TgAb}$ titre to rebound, and more postpartum data obtained at time points that are more distant from the time of delivery are needed to analyse the rebound of $\operatorname{TgAb}$.

With regard to TRAbs, in a study from Japan, researchers used four methods (first-, second-, and third-generation TBII assays and a bioassay) to measure serum TRAb levels in 23 women during pregnancy. They observed a decrease in both TRAbs and TSAbs during the progression of pregnancy, independent of the assay method used. ${ }^{16}$ The study of Amino $\mathrm{N}$ included six patients, and typical change patterns were observed in both TBII and TSAb assays in all patients. ${ }^{24}$ In the first part of our research, according to our analysis, in the TRAb group, the antibody titre dropped significantly from $\mathrm{T} 1$ to $\mathrm{T} 2$ in the LT4 subgroup, while statistical methods were not applicable to the nomedication subgroup. There was no significant difference between T2 and T3 or between T3 and PP in either subgroup. In the second part of our research, there were 3 patients in the LT4 subgroup and 1 patient in the nomedication subgroup, and the P-value per the Friedman

Table 2 Friedman Test for Patients with Complete Data for All 4 Periods

\begin{tabular}{|l|l|l|l|}
\hline & TgAbs & TRAbs & TPOAbs \\
\hline LT4 & 0.013 & 0.122 & $<0.001$ \\
No medication & 0.004 & & 0.272 \\
\hline
\end{tabular}

Abbreviations: $\operatorname{TgAbs}$, thyroid globulin antibodies; TRAbs, thyroid-stimulating hormone receptor antibodies; TPOAbs, thyroid peroxidase antibodies; LT4, levothyroxine. test was 0.122 in the LT4 subgroup. We thought these results may be due to the small sample size in the TRAb group (4 patients between $\mathrm{T} 2$ and $\mathrm{T} 3$ and 3 patients between T3 and PP in the LT4 subgroup; 2 patients between $\mathrm{T} 1$ and $\mathrm{T} 2,3$ patients between $\mathrm{T} 2$ and $\mathrm{T} 3$, and 4 patients between T3 and PP in the no-medication subgroup), which might lead to a relatively large statistical bias. One reason for the small sample size is that according to the 2019 Guidelines of the Chinese Society of Endocrinology and Chinese Society of Perinatal Medicine for the Diagnosis and Management of Thyroid Disease During Pregnancy, if the serum TRAb titre is negative in $\mathrm{T} 1$, it does not need to be tested again during pregnancy. Therefore, the sample size for the TRAb assessment was not as high as that for either the TPOAb or TgAb assessment.

For TPOAbs, in a recent study including forty TPOAbpositive euthyroid women, the average serum TPOAb levels were $209 \pm 284.32 \mathrm{IU} / \mathrm{mL}, 112.34 \pm 126.08 \mathrm{IU} / \mathrm{mL}$, $76.06 \pm 83.44 \mathrm{IU} / \mathrm{mL}$, and $84.13 \pm 106.32 \mathrm{IU} / \mathrm{mL}$ in the first, second, and third trimesters of pregnancy and in the PP (6 weeks after delivery), respectively. The TPOAb titre decreased significantly in the second and third trimesters compared to that in the first trimester $(\mathrm{p}=0.016$ and 0.003 , respectively). ${ }^{17}$ According to a study by A.B. PARKES, the average TPOAb level increased from $20 \mathrm{KIU} / \mathrm{L}$ to 121 $\mathrm{KIU} / \mathrm{L}$ throughout the postpartum year in the euthyroid TPOAb-positive group. In the PPT group, the TPOAb level reached the peak level of $181 \mathrm{KIU} / \mathrm{L}$ five months postpartum, compared to $28 \mathrm{KIU} / \mathrm{L}$ at delivery, and dropped to $118 \mathrm{KIU} / \mathrm{L}$ by twelve months postpartum. ${ }^{25}$ 

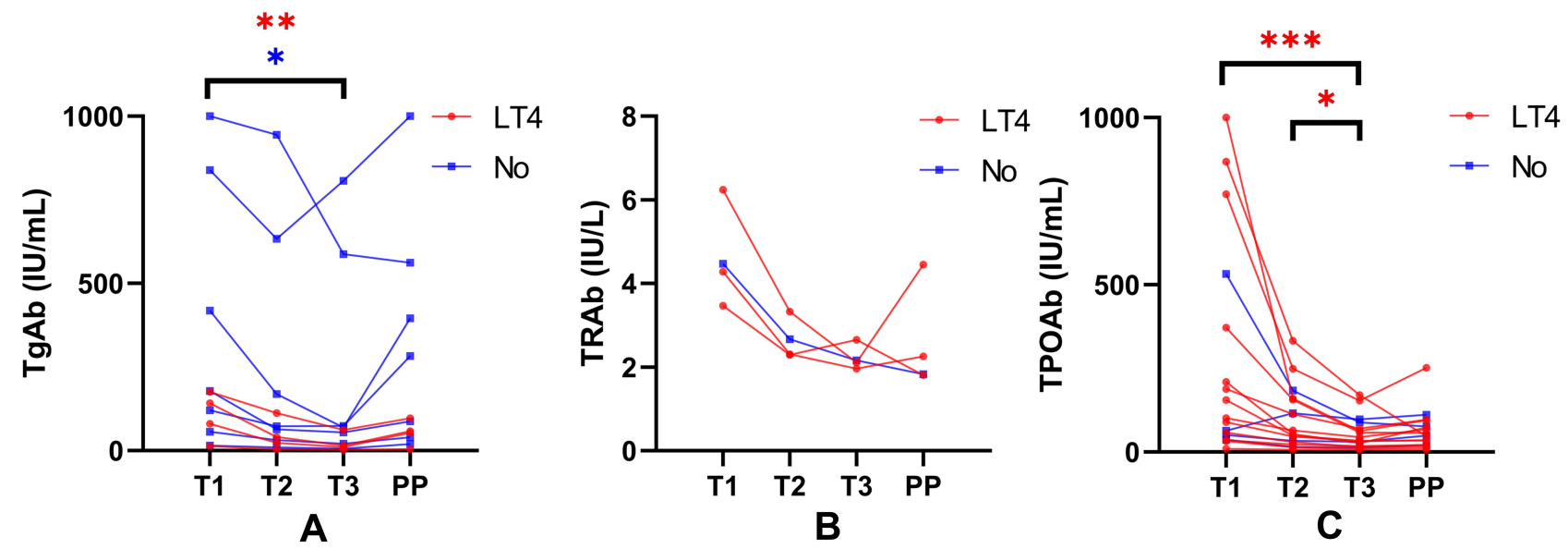

Figure 5 The variation in antibodies in patients who had complete laboratory examination results in all four periods. The three figures are data from patients who had complete laboratory examination results in four periods. (A) The results of 4 patients with LT4 treatment and 7 patients with no medication in the TgAb group. (B) The results of 3 patients with LT4 treatment and I patient with no medication in the TRAb group. (C) The results of I3 patients with LT4 treatment and 4 patients with no medication in the TPOAb group. $* 0.0 \mathrm{I}<\mathrm{P}<0.05 ; * 0.00 \mathrm{I}<\mathrm{P} \leq 0.0 \mathrm{I} ; * * * \mathrm{P} \leq 0.00 \mathrm{I}$.

Abbreviations: LT4, levothyroxine; No, no medication; ns, no significant difference.

In the first part of our study, according to our analysis, the antibody titre dropped significantly during pregnancy in both subgroups and rebounded significantly after delivery in the LT4 subgroup, but there was no significant difference in the titre between the T3 and PP periods in the nomedication subgroup. In the second part, significant differences were found only between $\mathrm{T} 2$ and $\mathrm{T} 3(0.014)$ and between $\mathrm{T} 1$ and $\mathrm{T} 3(\mathrm{P}<0.001)$ in the LT4 subgroup. We think the reasons for the lack of significant differences between T3 and PP in the no-medication subgroup are early postpartum testing at 12 weeks, and some women whose levels peak later may have been missed. ${ }^{25,26}$ In the second part of our study, the persuasiveness of the data was limited due to the small number of patients in the no-medication subgroup (4 patients) compared to the number of patients in the LT4 subgroup (13 patients).

Some of the patients had used drugs for GD and HT, which might have affected the antibody titres. LT4 has been used to treat hypothyroidism for many years. ${ }^{27}$ However, although reported, there is no consensus on how LT4 can influence antibody titres. Hashizume et al found that the use of FT4 during pregnancy and postpartum might decrease TRAb levels and protect women from the recurrence of hyperthyroidism after delivery compared with no drug use. ${ }^{28}$ Researchers have found that LT4 might suppress TSH and TPOAb levels in Hashimoto's thyroiditis patients. ${ }^{29}$ In the study of Nalan Okuroglu, they found a low but statistically significant positive relationship between the TPOAb $(r=0.217, \mathrm{P}<0.01)$ and $\mathrm{TgAb}$ levels $(\mathrm{r}=0.158, \mathrm{P}<0.05)$ and LT4 doses in the antibody-positive group; however, the involved patients were already undergoing replacement therapy, which means their antibody titres were not tested before LT4 treatment. ${ }^{30}$ In addition, it is impossible to determine whether LT4 treatment caused a high antibody titre or whether a high antibody titre caused low FT4 and high TSH, so a larger dose of LT4 is needed. In a study by OLLE KAMPE, LT4 $(0.1 \mathrm{mg})$ reduced the degree of hypothyroidism but had no detectable influence on the changes in TPOAb titres in the postpartum period. ${ }^{29}$ 1. Chiovato found that LT4 therapy induces a decrease in thyroid microsomal antibody (TPOAb) and TgAb in idiopathic myxoedema and in hypothyroid but not in euthyroid Hashimoto's thyroiditis. ${ }^{31}$ In the study by ROLF JANSSON, ten hypothyroid patients with Hashimoto's thyroiditis were treated with methimazole $30 \mathrm{mg}$ in addition to thyroxine $0.15 \mathrm{mg}$ daily. Another 10 hypothyroid patients with Hashimoto's thyroiditis were given thyroxine $0.15 \mathrm{mg}$ alone. After 22 weeks of treatment, significant decreases in thyroid microsomal autoantibody (TPOAb) titres were observed in both groups ( $\mathrm{p}<0.01)$; however, there was no difference in the mean change in titre between the two groups. When the patients treated with methimazole were subsequently given thyroxine $0.15 \mathrm{mg}$ alone for an additional 22 weeks, no additional change in titre was observed. ${ }^{32}$ Therefore, we think it is difficult to judge the effect of LT4 on antibody titre. PTU and methimazole (MMI) are commonly used antithyroid drugs for the treatment of hyperthyroidism. However, PTU is suggested to be used in the first trimester, and MMI is suggested to be used in the second and third trimesters. ${ }^{33,34}$ According to 
research by Tsai et al, although there was no significant difference in the TRAb levels between the PTU/propranolol combination therapy and the PTU/cholestyramine/propranolol combination therapy in the treatment of Graves' hyperthyroidism, both treatment combinations could lead to a decrease in TRAbs during the study period. ${ }^{35}$ ChihTsuengHe et al observed a decrease in TRAbs in both the MMI and PTU treatment groups. ${ }^{36}$ Momotani et al observed a decrease in TBII in infants who were receiving nutrients only via breastfeeding from mothers who took PTU at a dose of $300 \mathrm{mg}$ daily. ${ }^{37}$ The results of studies on the effect of PTU on TRAb are relatively consistent. It is believed that both PTU and MMI can lead to a decrease in TRAb titre, which means that hyperthyroidism is relieved. ${ }^{36-40}$ Women should conceive when they are in the euthyroid state. For women with hyperthyroidism before conception, ablative therapy (131I or surgery) or medical therapy is recommended. For women with a high TRAb titre who wish to become pregnant, surgery is recommended since the TRAb titre will increase and remain at a high level after 131I ablation therapy. ${ }^{41}$ We must mention that some of our patients underwent selenium therapy; this it is not recommended for women during pregnancy. ${ }^{33} \mathrm{We}$ did not record the specific dose of selenium used, and the effects of selenium on TRAbs, TPOAbs and TgAbs are controversial. Several studies have reported that selenium can lead to a decrease in antithyroid antibodies, while some have shown that selenium has no effect on antibody titre. The conditions of both pregnant women and the general population were included in these studies, as were all three antibody types. ${ }^{15,33,42-50}$ Thus, the effects of selenium on the antibody titre cannot be determined at present. According to previous studies, drugs may influence antibody titres. Therefore, the effect of selenium needs to be further studied. In addition to drugs, both the impact of adherence by endocrinologists to guidelines regarding LT4 therapy and patient compliance with the management of hypothyroidism has been studied, specifically by Vezzani et al. ${ }^{51}$ They found that the concordance rates of Italian endocrinologists with American Association of Clinical Endocrinologists/American Thyroid Association (AACE/ ATA) and European Thyroid Association (ETA) recommendations were $77.1 \%$ and $71.7 \%$, respectively. Patients with high compliance were unlikely to have well-controlled hypothyroidism. However, the association between the adherence of doctors to guidelines and variations in antibodies has not been reported. In our research, as all patients were treated by Dr. Guan Liang, the problem of different levels of adherence to guidelines among different doctors was avoided. Although we did not use the Morisky-Green Medical Adherence Scale-8 (MMAS-8) scale to study patient compliance with the treatment scheme, according to the results of Vezzani et al, we thought that different compliance rates of patients might not affect the variations in antibodies.

The limitations of other studies are their lack of inclusion of all three antibody types in one study. In addition, most of the studies used the reference level of antibodies, and the decrease in antibody titre was reflected by the decrease in the positive rate. The advantages of our research are our inclusion all three antibody types and our use of different methods to determine the P-values during different periods of pregnancy. Thus, the variations in the three antibody types could be reflected simultaneously. The results are shown as a decrease in titre rather than the positive rate in our research, avoiding the loss of information when the titre is converted to the positive rate. We separated our patients into different groups according to the medication they had, thus eliminating the influence of medication on antibody titre. However, the disadvantages of our research are that our sample size was small, and that not all patients had complete data in each period. Due to the explosion of coronavirus in China in 2020, public transportation could not function at normal levels, causing many patients to not come to Ruijin Hospital to have their antibody titres measured. Our results need to be supported by a larger group of subjects from a larger number of medical centres. In addition, since many patients did not have TRAb tests, the P-value in the TRAb group may not be accurate. All the patients were restricted to Dr. Guan Liang; thus, selection bias may have occurred.

At present, the most important explanation is that the decrease in the antithyroid antibody titre is caused by Tregmediated suppression. ${ }^{52}$ Regulatory $\mathrm{CD} 4{ }^{+} \mathrm{CD} 25^{+} \mathrm{T}$ cells (Tregs) are important for peripheral tolerance against both foreign and self-antigens and in foetal tolerance. Tregs can regulate Th1-type cellular immune activity and Th2-type humoural immune activity. ${ }^{53}$ Once a woman becomes pregnant, foetal antigens and oestrogen will provoke the production of Tregs, which will increase during pregnancy and peak in the second trimester. Then, the reactions of Th1 and Th2 cells against paternal/foetal alloantigens are suppressed by Tregs. Thus, the antithyroid antibody titre decreases during pregnancy. ${ }^{19,54}$ Tregs may also migrate towards the feto-maternal interface and then generate a series of tolerant environments, such as TGF- $\beta$, leukaemia, inhibitory factor and HO1, which may help protect the 
process of pregnancy. ${ }^{15}$ The data of Nobuyuki showed that both Th1 and Th2-type cytokines decreased during pregnancy, although both immune reactions increased after delivery, ${ }^{24}$ which supports this point of view. Dysfunction of Tregs or deficiency in proliferative responsiveness may endanger pregnancy. ${ }^{15}$ After delivery, the number of Tregs returns to prepregnancy levels, which then leads to the exacerbation of AITDs. ${ }^{23,55}$ MHC and B cells also play a part in immunosuppression during pregnancy. Since the villous trophoblasts and syncytiotrophoblast microparticles in the feto-maternal interface lack HLA class I and class II proteins, they express only MHC molecules such as HLA-S, $-\mathrm{E},-\mathrm{F}$ and $-\mathrm{G},{ }^{56}$ which may resist NK cells. ${ }^{57} \mathrm{~B}$ cells are antigen-presenting cells that can interact with T-cell receptors. During pregnancy, the number of B cells in the circulation will not considerably change, but as Tregs increase, the autoantibody titre decreases. Oestrogen is also thought to be able to pass negative signals to $\mathrm{B}$ cells during pregnancy. ${ }^{58}$

\section{Conclusions}

According to the data we collected and analysed, we can conclude that the titres of TgAbs and TPOAbs decrease during pregnancy, whether under the use of LT4 or not. At present, there is only one set of reference levels of these antibodies during pregnancy. We advocate for determining standard, or normal, levels at different periods during pregnancy for comparisons of variations in these antibodies. We suggest that patients have regular check-ups with their doctors to avoid the adverse outcomes associated with abnormal elevations in antithyroid antibodies.

\section{Acknowledgments}

We acknowledge the work of Xuemin Qiu, Lisha Li, Yanzhi Zhang, Lan Wang, Yizhen Sima, and Lijuan Li, who have not been listed as authors of this article.

\section{Disclosure}

The authors declare that there are no conflicts of interest regarding the publication of this article.

\section{References}

1. Matthews DC, Syed AA. The role of TSH receptor antibodies in the management of Graves' disease. Eur J Intern Med. 2011;22(3): 213-216. doi:10.1016/j.ejim.2011.02.006

2. Hollowell JG, Staehling NW, Flanders WD, et al. Serum TSH, T(4), and thyroid antibodies in the United States population (1988 to 1994): national health and nutrition examination survey (NHANES III) J Clin Endocrinol Metab. 2002;87(2):489-499. doi:10.1210/jcem.87. 2.8182
3. Chen X, Jin B, Xia J, et al. Effects of thyroid peroxidase antibody on maternal and neonatal outcomes in pregnant women in an iodine-sufficient area in China. Int $J$ Endocrinol. 2016;2016:64 61380. doi:10.1155/2016/6461380

4. Bucci I, Giuliani C, Napolitano G. Thyroid-stimulating hormone receptor antibodies in pregnancy: clinical relevance. Front Endocrinol (Lausanne). 2017;8:137. doi:10.3389/fendo.2017.00137

5. Li Y, Xu T, Mo Q, Fu W, Yao C. Thyrotropin receptor antibody: a novel risk indicator for pregnancy loss. Clin Biochem. 2019;64:44-48. doi:10.1016/j.clinbiochem.2018.11.017

6. De Leo S, Pearce EN. Autoimmune thyroid disease during pregnancy. Lancet Diabetes Endocrinol. 2018;6(7):575-586. doi:10. 1016/S2213-8587(17)30402-3

7. Rajput R, Yadav T, Seth S, Nanda S. Prevalence of thyroid peroxidase antibody and pregnancy outcome in euthyroid autoimmune positive pregnant women from a tertiary care center in Haryana. Indian J Endocrinol Metab. 2017;21(4):577-580. doi:10.4103/ijem. IJEM $397 \quad 16$

8. Korevaar TI, Derakhshan A, Taylor PN, et al. Association of thyroid function test abnormalities and thyroid autoimmunity with preterm birth: a systematic review and meta-analysis. JAMA. 2019;322(7): 632-641. doi:10.1001/jama.2019.10931

9. van Dijk MM, Smits IH, Fliers E, Bisschop PH. Maternal thyrotropin receptor antibody concentration and the risk of fetal and neonatal thyrotoxicosis: a systematic review. Thyroid. 2018;28(2):257-264. doi:10.1089/thy.2017.0413

10. Han Y, Mao LJ, Ge X, et al. Impact of maternal thyroid autoantibodies positivity on the risk of early term birth: Ma'anshan Birth Cohort Study. Endocrine. 2018;60(2):329-338. doi:10.1007/s12020-018-15 76-6

11. Wang X, Liu H, Zhang Y, et al. Effects of isolated positive maternal thyroglobulin antibodies on brain development of offspring in an experimental autoimmune thyroiditis model. Thyroid. 2015;25(5): 551-558. doi:10.1089/thy.2014.0310

12. Dhillon-Smith RK, Coomarasamy A. Levothyroxine in women with thyroid peroxidase antibodies before conception REPLY. New Engl $J$ Med. 2019;381(2):190-191.

13. Nazarpour S, Ramezani Tehrani F, Simbar M, et al. Effects of levothyroxine on pregnant women with subclinical hypothyroidism, negative for thyroid peroxidase antibodies. J Clin Endocrinol Metab. 2018;103(3):926-935. doi:10.1210/jc.2017-01850

14. Abeillon-du Payrat J, Chikh K, Bossard N, et al. Predictive value of maternal second-generation thyroid-binding inhibitory immunoglobulin assay for neonatal autoimmune hyperthyroidism. Eur J Endocrinol. 2014;171(4):451-460. doi:10.1530/EJE-14-0254

15. Balucan FS, Morshed SA, Davies TF. Thyroid autoantibodies in pregnancy: their role, regulation and clinical relevance. $J$ Thyroid Res. 2013;2013:182472. doi:10.1155/2013/182472

16. Kamijo K. TSH-receptor antibodies determined by the first, second and third generation assays and thyroid-stimulating antibody in pregnant patients with Graves' disease. Endocr J. 2007;54(4):619-624. doi:10.1507/endocrj.K06-196

17. Meena M, Chopra S, Jain V, Aggarwal N. The effect of anti-thyroid peroxidase antibodies on pregnancy outcomes in euthyroid women. J Clin Diagn Res. 2016;10(9):QC04-QC07. doi:10.7860/JCDR/2016/ 19009.8403

18. Han Y, Mao LJ, Ge X, et al. Thyroid autoantibodies in pregnancy are associated with hypertensive disorders of pregnancy: Ma'anshan Birth cohort study. Clin Endocrinol. 2018;88(6):928-935. doi:10.1111/ cen. 13590

19. Stagnaro-Green A, Roman SH, Cobin RH, el-Harazy E, Wallenstein S, Davies TF. A prospective study of lymphocyte-initiated immunosuppression in normal pregnancy: evidence of a T-cell etiology for postpartum thyroid dysfunction. J Clin Endocrinol Metab. 1992;74 (3):645-653. doi:10.1210/jcem.74.3.1740500 
20. Tamaki H, Katsumaru H, Amino N, Nakamoto H, Ishikawa E, Miyai K. Usefulness of thyroglobulin antibody detected by ultrasensitive enzyme immunoassay: a good parameter for immune surveillance in healthy subjects and for prediction of post-partum thyroid dysfunction. Clin Endocrinol (Oxf). 1992;37(3):266-273. doi:10.11 11/j.1365-2265.1992.tb02321.x

21. Amino N, Kuro R, Tanizawa O, et al. Changes of serum anti-thyroid antibodies during and after pregnancy in autoimmune thyroid diseases. Clin Exp Immunol. 1978;31(1):30-37.

22. D'Armiento M, Salabe H, Vetrano G, Scucchia M, Pachi A. Decrease of thyroid antibodies during pregnancy. $J$ Endocrinol Invest. 1980;3 (4):437-438. doi:10.1007/BF03349385

23. Smyth PP, Wijeyaratne CN, Kaluarachi WN, et al. Sequential studies on thyroid antibodies during pregnancy. Thyroid. 2005;15(5): 474-477. doi:10.1089/thy.2005.15.474

24. Amino N, Izumi Y, Hidaka Y, et al. No increase of blocking type antithyrotropin receptor antibodies during pregnancy in patients with Graves' disease. J Clin Endocr Metab. 2003;88(12):5871-5874. doi:10.1210/jc.2003-030971

25. Parkes AB, Othman S, Hall R, John R, Richards CJ, Lazarus JH. The role of complement in the pathogenesis of postpartum thyroiditis. $J$ Clin Endocrinol Metab. 1994;79(2):395-400. doi:10.1210/jcem. 79.2.8045954

26. Ekinci EI, Chiu WL, Lu ZX, et al. A longitudinal study of thyroid autoantibodies in pregnancy: the importance of test timing. Clin Endocrinol (Oxf). 2015;82(4):604-610. doi:10.1111/cen.12571

27. Jonklaas J, Bianco AC, Bauer AJ, et al. Guidelines for the treatment of hypothyroidism: prepared by the american thyroid association task force on thyroid hormone replacement. Thyroid. 2014;24(12): 1670-1751. doi:10.1089/thy.2014.0028

28. Hashizume K, Ichikawa K, Nishii Y, et al. Effect of administration of thyroxine on the risk of postpartum recurrence of hyperthyroid Graves' disease. J Clin Endocrinol Metab. 1992;75(1):6-10. doi:10.1210/jcem.75.1.1642700

29. Kampe O, Jansson R, Karlsson FA. Effects of L-thyroxine and iodide on the development of autoimmune postpartum thyroiditis. $J$ Clin Endocrinol Metab. 1990;70(4):1014-1018. doi:10.1210/jcem-70-4-1014

30. Okuroglu N, Ozdemir A, Sertbas Y, Sancak S. The relationship between thyroid antibody titer and levothyroxine dose in patients with overt primary hypothyroidism. Ann Saudi Med. 2017;37 (3): 189-193. doi:10.5144/0256-4947.2017.189

31. Chiovato L, Marcocci C, Mariotti S, Mori A, Pinchera A. L-thyroxine therapy induces a fall of thyroid microsomal and thyroglobulin antibodies in idiopathic myxedema and in hypothyroid, but not in euthyroid Hashimoto's thyroiditis. J Endocrinol Invest. 1986;9 (4):299-305. doi:10.1007/BF03346932

32. Jansson R, Karlsson A, Dahlberg PA. Thyroxine, methimazole, and thyroid microsomal autoantibody titres in hypothyroid Hashimoto's thyroiditis. Br Med J (Clin Res Ed). 1985;290(6461):11-12. doi:10. $1136 / \mathrm{bmj} .290 .6461 .11$

33. Stagnaro-Green A, Abalovich M, Alexander E, et al. Guidelines of the American Thyroid Association for the diagnosis and management of thyroid disease during pregnancy and postpartum. Thyroid. 2011;21(10):1081-1125. doi:10.1089/thy.2011.0087

34. Bartalena L, Burch HB, Burman KD, Kahaly GJA. 2013 European survey of clinical practice patterns in the management of Graves' disease. Clin Endocrinol (Oxf). 2016;84(1):115-120. doi:10.1111/ cen. 12688

35. Tsai WC, Pei D, Wang TF, et al. The effect of combination therapy with propylthiouracil and cholestyramine in the treatment of Graves' hyperthyroidism. Clin Endocrinol (Oxf). 2005;62(5):521-524. doi:10. 1111/j.1365-2265.2005.02249.x

36. He CT, Hsieh AT, Pei D, et al. Comparison of single daily dose of methimazole and propylthiouracil in the treatment of Graves' hyperthyroidism. Clin Endocrinol (Oxf). 2004;60(6):676-681. doi:10. $1111 / \mathrm{j} .1365-2265.2004 .02032 . x$
37. Momotani N, Yamashita R, Makino F, Noh JY, Ishikawa N, Ito K. Thyroid function in wholly breast-feeding infants whose mothers take high doses of propylthiouracil. Clin Endocrinol (Oxf). 2000;53 (2):177-181. doi:10.1046/j.1365-2265.2000.01078.x

38. Teng CS, Yeung RT. Changes in thyroid-stimulating antibody activity in Graves' disease treated with antithyroid drug and its relationship to relapse: a prospective study. J Clin Endocrinol Metab. 1980;50 (1):144-147. doi:10.1210/jcem-50-1-144

39. Takasu N, Yamashiro K, Komiya I, Ochi Y, Sato Y, Nagata A. Remission of Graves' hyperthyroidism predicted by smooth decreases of thyroid-stimulating antibody and thyrotropin-binding inhibitor immunoglobulin during antithyroid drug treatment. Thyroid. 2000;10(10):891-896. doi:10.1089/thy.2000.10.891

40. Feldt-Rasmussen U, Bech K, Date J, Hyltoft Pedersen P, Johansen K, Nistrup Madsen S. Thyroid stimulating antibodies, thyroglobulin antibodies and serum proteins during treatment of Graves' disease with radioiodine or propylthiouracil. Allergy. 1982;37(3):161-167. doi:10.1111/j.1398-9995.1982.tb01892.x

41. Laurberg P, Bournaud C, Karmisholt J, Orgiazzi J. Management of Graves' hyperthyroidism in pregnancy: focus on both maternal and foetal thyroid function, and caution against surgical thyroidectomy in pregnancy. Eur J Endocrinol. 2009;160(1):1-8. doi:10.1530/EJE-080663

42. McLachlan SM, Aliesky H, Banuelos B, Hee SSQ, Rapoport B. Variable effects of dietary selenium in mice that spontaneously develop a spectrum of thyroid autoantibodies. Endocrinology. 2017;158(11):3754-3764. doi:10.1210/en.2017-00275

43. Mazokopakis EE, Papadakis JA, Papadomanolaki MG, et al. Effects of 12 months treatment with L-selenomethionine on serum anti-TPO levels in patients with Hashimoto's thyroiditis. Thyroid. 2007;17 (7):609-612. doi:10.1089/thy.2007.0040

44. Mantovani G, Isidori AM, Moretti C, et al. Selenium supplementation in the management of thyroid autoimmunity during pregnancy: results of the "SERENA study", a randomized, double-blind, placebo-controlled trial. Endocrine. 2019;66(3):542-550. doi:10.1007/ s12020-019-01958-1

45. Kyrgios I, Giza S, Kotanidou EP, et al. 1-selenomethionine supplementation in children and adolescents with autoimmune thyroiditis: a randomized double-blind placebo-controlled clinical trial. J Clin Pharm Ther. 2019;44(1):102-108. doi:10.1111/jcpt.12765

46. Krysiak R, Szkrobka W, Okopien B. Atorvastatin potentiates the effect of selenomethionine on thyroid autoimmunity in euthyroid women with Hashimoto's thyroiditis. Curr Med Res Opin. 2019;35 (4):675-681. doi:10.1080/03007995.2018.1541314

47. Krysiak R, Szkrobka W, Okopien B. The effect of vitamin D and selenomethionine on thyroid antibody titers, hypothalamic-pituitarythyroid axis activity and thyroid function tests in men with Hashimoto's thyroiditis: a pilot study. Pharmacol Rep. 2019;71 (2):243-247. doi:10.1016/j.pharep.2018.10.012

48. Gartner R, Gasnier BC, Dietrich JW, Krebs B, Angstwurm MW. Selenium supplementation in patients with autoimmune thyroiditis decreases thyroid peroxidase antibodies concentrations. J Clin Endocrinol Metab. 2002;87 (4):1687-1691. doi:10.1210/jcem.87.4.8421

49. Fan Y, Xu S, Zhang H, et al. Selenium supplementation for autoimmune thyroiditis: a systematic review and meta-analysis. Int J Endocrinol. 2014;2014:904573. doi:10.1155/2014/904573

50. Dharmasena A. Selenium supplementation in thyroid associated ophthalmopathy: an update. Int J Ophthalmol. 2014;7(2):365-375. doi:10.3980/j.issn.2222-3959.2014.02.31

51. Vezzani S, Giannetta E, Altieri B, et al. An Italian survey of compliance with major guidelines for 1-thyroxine of primary hypothyroidism. Endocr Pract. 2018;24(5):419-428. doi:10.4158/EP-2017-0159

52. Gaberscek S, Zaletel K. Thyroid physiology and autoimmunity in pregnancy and after delivery. Expert Rev Clin Immunol. 2011;7(5):697-706; quiz 707. doi:10.1586/eci.11.42 
53. Saito S, Nakashima A, Shima T, Ito M. Th1/Th2/Th17 and regulatory T-cell paradigm in pregnancy. Am J Reprod Immunol. 2010;63 (6):601-610. doi:10.1111/j.1600-0897.2010.00852.x

54. Weetman AP. Immunity, thyroid function and pregnancy: molecular mechanisms. Nat Rev Endocrinol. 2010;6(6):311-318. doi:10.1038/ nrendo. 2010.46

55. Chan GW, Mandel SJ. Therapy insight: management of Graves' disease during pregnancy. Nat Clin Pract Endocrinol Metab. 2007;3 (6):470-478. doi:10.1038/ncpendmet0508

56. Tilburgs T, Scherjon SA, Claas FHJ. Major histocompatibility complex (MHC)-mediated immune regulation of decidual leukocytes at the fetal-maternal interface. $J$ Reprod Immunol. 2010;85(1):58-62. doi:10.1016/j.jri.2010.01.005
57. Favier B, LeMaoult J, Rouas-Freiss N, Moreau P, Menier C, Carosella ED. Research on HLA-G: an update. Tissue Antigens. 2007;69(3):207-211. doi:10.1111/j.1399-0039.2006.00757.x

58. Kincade PW, Medina KL, Smithson G, Scott DC. Pregnancy: a clue to normal regulation of B lymphopoiesis. Immunol Today. 1994;15 (11):539-544. doi:10.1016/0167-5699(94)90211-9

\section{Publish your work in this journal}

Risk Management and Healthcare Policy is an international, peerreviewed, open access journal focusing on all aspects of public health, policy, and preventative measures to promote good health and improve morbidity and mortality in the population. The journa welcomes submitted papers covering original research, basic science, clinical \& epidemiological studies, reviews and evaluations, guidelines, expert opinion and commentary, case reports and extended reports. The manuscript management system is completely online and includes a very quick and fair peer-review system, which is all easy to use. Visit http://www.dovepress.com/testimonials.php to read real quotes from published authors.

Submit your manuscript here: https://www.dovepress.com/risk-management-and-healthcare-policy-journal 